\title{
Review
}

\section{The Design of Scaffolds for Use in Tissue Engineering. Part II. Rapid Prototyping Techniques}

\author{
SHOUfEng YANG, Ph.D., KAH-FAI LEONG, M.S.E., M.S.M.E., ZHAOHUI DU, Ph.D., \\ and CHEE-KAI CHUA, Ph.D.
}

\begin{abstract}
Tissue engineering (TE) is an important emerging area in biomedical engineering for creating biological alternatives for harvested tissues, implants, and prostheses. In TE, a highly porous artificial extracellular matrix or scaffold is required to accommodate mammalian cells and guide their growth and tissue regeneration in three-dimension (3D). However, existing 3D scaffolds for TE proved less than ideal for actual applications because they lack mechanical strength, interconnected channels, and controlled porosity or pores distribution. In this paper, the authors review the application and advancement of rapid prototyping (RP) techniques in the design and creation of synthetic scaffolds for use in TE. We also review the advantages and benefits, and limitations and shortcomings of current RP techniques as well as the future direction of RP development in TE scaffold fabrication.
\end{abstract}

\section{INTRODUCTION}

\begin{abstract}
A S DISCUSSED in Part I (Tissue Engineering 7, 679-689, 2001) of this two-part paper, approximately onequarter of patients in need of organ transplants in the United States die while waiting for a suitable donor. ${ }^{1,2}$ The current demands for transplant organs and tissues far outpace supply, and all manner of projections indicate that this gap will continue to widen. ${ }^{1,3}$ Cell transplantation is recently proposed as an alternative treatment to whole organ transplantation for failing or malfunctioning organs. ${ }^{4-6}$ For the creation of an autologous implant, donor tissue is harvested and dissociated into individual cells, and the cells are attached and cultured onto a proper substrate that is ultimately implanted back at the desired site of the functioning tissue. Because many isolated cell populations can be expanded in vitro using cell culture techniques, only a very small number of donor cells may be needed to prepare such implants. However, it is believed that isolated cells cannot form new tissues by themselves. Most primary organ cells are believed to be anchorage-dependent and require specific environments that very often include the presence of a sup-
\end{abstract}

Design Research Center, School of Mechanical and Production Engineering, Nanyang Technological University, Singapore. 
porting material to act as a template for growth. The success of any cell transplantation therapy relies on the development of suitable substrates for both in vitro and in vivo tissue culture. Existing substrates, mainly in the form of tissue engineering scaffold, are considered to be less than ideal for applications because they lack mechanical strength, interconnected channels, and controlled porosity or pores distribution.

Rapid prototyping (RP), also termed "solid freeform fabrication (SFF)," is a recent technology based on the advanced development of computer and manufacturing. The main advantage of these techniques is their ability to produce complex products rapidly directly from a computer model. RP has been used in the medical field primarily as a means of guiding surgical procedures using tactile models derived from patient computerized tomography (CT) data. ${ }^{7}$ These models have also been used to cast custom titanium orbital implants. Direct fabrication of custom implants is promising in offering simpler and more rapid surgical implementations. The potential to intimately control the microstructure of porous channels and the overall macroscopic shape of the implants makes RP an ideal process for fabricating implant and tissue engineering scaffold as well. In this paper, we review the applications and advancement of RP techniques in the design and creation of synthetic scaffolds for use in tissue engineering (TE). Part I of this paper $^{8}$ analyzed the factors necessary to enhance the design and creation of scaffolds for use in TE in terms of materials, structure, and mechanical properties. Based on these requirements, the authors will now further discuss the advantages and benefits, and limitations and shortcomings of current RP techniques, as well as the future direction of application of RP in fabricating TE scaffolds.

\section{EXISTING APPLICATIONS OF RAPID PROTOTYPING IN TISSUE ENGINEERING}

Traditional fabrication methods cannot build parts with predefined or controlled microstructure as well as macrostructure. RP, or SFF, has the distinct advantage of being able to build objects with predefined macrostructures as well as microstructures. This distinct advantage makes RP a technique with excellent potential for fabricating scaffold with controlled hierarchical structures for use in TE. The macroscopic shape of the scaffold, on a scale of up to tens of millimeters, will determine the external appearance and structure of the final product. For example, it may be desirable to reconstruct an ear or a jaw exactly to meet patient contours based on images acquired using magnetic resonance imaging (MRI) or CT scans directly from the patient. The size, orientation, and surface chemistry of pores and channels, on a scale of hundreds of microns, have a direct impact on the extent and nature of tissue in-growth. On the scale of tens of microns, local surface texture and porosity become important.

There are also two particular characteristics of RP systems that limits their performance. One is the overall resolution of the processes achievable in current systems and the second is the ranging materials from which prototypes are made. Several different RP processes are described in the following sections. Their typical features, characteristics and processing limitations with regard to TE are highlighted.

\section{Sheet lamination}

Sheet lamination fabrication ${ }^{7,9}$ such as the patented laminated object manufacturing (LOM) process builds three-dimensional (3D) cross-sections out of a roll of sheets lined with thermoplastic adhesive. Layered cross-sectional profiles are cut with a $\mathrm{CO}_{2}$ laser and by bonding each cross-sectional layer to the previous one by applying heat and pressure. The prototype is sequentially made layer by layer, and the area outside the layer outline and inside any internal closed areas that are not part of the profile are cut into small sections called "tiles." These tiles are removed in a postprocessing phase after the completion of all the layers that comprise the object. Small features, in particular small inner holes, cannot be fabricated by this method.

Steidle et al. ${ }^{10,11}$ have reported the use of a nonresorbable bioceramic composite system consisting of hydroxyapatite particles bonded together by a calcium phosphate glass phase to build a biocompatible bone for implant. The implant built is almost completely dense, which renders it unsuitable for use in its intended application in bone tissue engineering. Although this kind of process has an advantage in its ability of recreating the external shape of the scaffold, it unfortunately still suffers the limitation of a lack of microstructure control, because the microstructure of each sheet is uniform. 


\section{Adhesion bonding}

In this method, 3D parts are created by a layered printing process with adhesive bonding, using powder as the base material, according to sliced cross-sectional computer-assisted design (CAD) data of the object. Each layer of powder is selectively joined where the part is to be formed by ink-jet printing of a binder material. The process is repeated layer by layer until the part is complete. An example of such technology is three-dimensional printing (3DP).

Cima et al. ${ }^{12,13}$ demonstrated using 3DP to build drug delivery devices and tissue regeneration devices. Although only polyethylene oxide (PEO) and polycaprolactone (PCL) powders were used in those experiments, theoretically and virtually any materials that can be processed into powdered form can be used for 3D printing. ${ }^{13}$ TheriForm ${ }^{\mathrm{TM}},{ }^{14}$ one of six licensees of 3DP, developed pharmaceutical dosage forms and medical products, including drug delivery and TE, based on 3DP fabrication process. Cima ${ }^{15}$ produced 200$\mu \mathrm{m}$ lines by printing polymer solutions rather than using pure solvent, while the later will produce a line primitive of $500 \mu \mathrm{m}$ in width.

In this method, adapting a appropriate binder for the different materials used can pose problems for biomedical applications. Some organic solvents that are in use now as binders, such as chloroform and methylene chloride, are harmful to the human body and are difficult to remove completely. Even after 1 week, during which samples of implants are placed in a vacuum to remove excess solvent, the amount of chloroform managed only to be reduced from $10 \mathrm{wt} \%$ to $0.5 \mathrm{wt} \%{ }^{16}$ Another difficulty is that excess powder that is trapped in small channels is difficult to remove. Also, channels of less than $1 \mathrm{~mm}$ in diameter have not been successfully built.

\section{Laser sintering}

In laser sintering methods, parts are built by sintering of powder on a powder bed, when an infrared laser beam hits a thin layer of powdered material such as wax, polycarbonate, nylon, or even metal. The interaction of the laser beam with the powder raises the local surface temperature to the glass transition temperature of the powder. This is just below the melting temperature. It results in particle bonding-fusing the particles onto each other and to the previous layer to form a solid. Limited by the power of the laser and thermal diffusion, the glass transition temperature and melting point of the powder cannot be too high. For ceramic powder, the polymer-ceramic mixture is always used, in which case the polymer is used as a low melting point binder.

Lee et al. ${ }^{17,18}$ coated calcium phosphate powder with polymer by spray drying slurry of particulate and emulsion binder. The coated powder was sintered by selective laser sintering (SLS) to fabricate artificial calcium phosphate bone implant. Postprocessing included infiltrating the sintered part with calcium phosphate solution or phosphoric acid-based inorganic cement. This was used to improve the density of green part to prevented collapse in the subsequently polymer binder burn out stage. However, the compressive strengths ${ }^{19}$ of the infiltrated sintered part without macropores was only $36.0 \pm 7.5 \mathrm{MPa}$. Implant model with macropores of about $2 \mathrm{~mm}$ in diameter was built for preclinical trials to assess the biocompatibility. It is believed that macropores in the implant should be smaller to increase the surface area for cell attachment and to improve the mechanical strength for load-bearing requirements.

The laser beam diameter of the Sinter Station 2500 (DTM, USA) is about $400 \mu \mathrm{m}$ (0.016 inches). Due to the Gaussian distribution of the laser energy and the nature of powder bonding, it becomes relatively difficult to form sharp corners and clear boundaries. The conduction and diffusion of laser heat cause neighboring powder of scan vector unwanted bonding, which can sometimes be serious (Fig. 1). These make it impossible to build small features of less than $400 \mu \mathrm{m}$ in size. The fuzzy boundary and the bonding powder induce a coarse inner surface on the macropores, and this makes it difficult to clean out the trapped unsintered powder.

Improvements on SLS process are expected to produce the desired scaffold for TE. These include acquiring the ability to create smaller features by using a smaller laser spot size, powder size, and thinner layer thickness. New ways have to be found to remove trapped loose powder. Potential solutions include using ultrasonic vibration, compressive air, bead blaster, and/or appropriate solvent. In addition, better sin- 

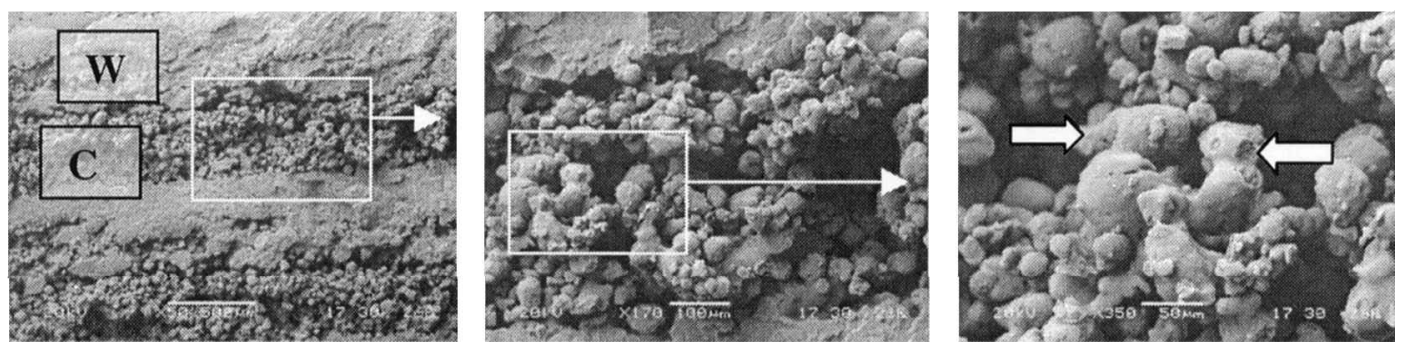

FIG. 1. Serious bonding of neighboring powder by laser heat diffusion in SLS (W, wall of channel, sintered part; C, channel, unsintered part). The arrows indicate the serious bonding of neighboring powder. Laser power: 5 W. Scan speed: $200 \mathrm{~m} / \mathrm{sec}$.

tering environment is necessary so that the humidity, inertness of surrounding atmosphere, and vacuum can be controlled within a smaller working chamber.

\section{Photopolymerization}

The principle of this method is based on the polymerization of photopolymer resins that is initiated by radiant energy from electromagnetic radiation. ${ }^{7}$ Photopolymer resins are mixtures of simple low-molecular-weight monomers capable of chain-reacting to form solid long-chain polymers when activated by radiant energy within specific wavelength range. There are two basic types of liquid-based commercial RP machines that use photopolymerization. One uses a laser, while the other uses a masked lamp to cure the photopolymers. ${ }^{7,9}$ In the first system, a deflected laser beam is used to irradiate a thin polymer layer at the surface of a vat filled with liquid photopolymer resin. The irradiated areas of photopolymer react chemically to become solid. For example, 3D system's stereolithography apparatus (SLA) uses an ultraviolet laser to solidify an epoxy resin. The second system uses masked illumination, instead of a point-by-point method used by laser system. It irradiates a complete layer of polymer every single time. An example of this masked lamp technique is Cubital's solid ground curing (SGC). Rapid micro product development (RMPD), ${ }^{20}$ a microstereolithography method developed by MicroTEC, and mentioned by Chua et al., ${ }^{9}$ is a promising RP approach. This technology, based on combination of masked lamp technology and laser curing photopolymerization, emerged in the middle of 1999.

Langton et al. ${ }^{21}$ described a method for the development of a user-defined structural model simulating cancellous bone of the human calcaneus using SLA. The potential for SLA-produced samples to be used as a structurally controlled cancellous bone mimic was investigated by producing a 3D rod lattice of $3 \mathrm{~mm}$ center-to-center distance and rod diameters of $1 \mathrm{~mm}$ (70\% porosity) representing healthy bone and $0.4 \mathrm{~mm}$ (95\% porosity) representing osteoporotic bone.

Chu et al. ${ }^{22}$ built hydroxyapatite prototypes for bone tissue scaffolds from Image-Based Design files, featuring an interior architecture of void passages. Direct ceramic SLA ${ }^{22}$ is done using UV-curable suspensions of ceramic powders in acrylates in a conventional SLA machine. Viscosity control for these highly concentrated suspensions and cure depth behavior are the main issues for fabricating a ceramic part with the stereolithography techniques.

Molecular Geodesics, Inc. (MGI, Boston, MA) ${ }^{23,24}$ developed a new class of biomimitic materials that mimic the microstructural organization, mechanical responsiveness, and biocatalytic activities of living cells and tissues. MGI's strategy is to study the underlying structures that provide living cells and tissues with their strength, flexibility, and porosity, and develop ways to apply these structures to synthetic products. A small-spot laser stereolithography system from 3D System is used. It has been demonstrated to be able to fabricate features as small as $70 \mu \mathrm{m}$, but the ideal feature required is less than half that size (Figs. 2-4).

The common laser spot size used in SLA is about $250 \mu \mathrm{m}$ in diameter. This is a result of a compromise between the demands of achieving precision and attaining maximum speed. However, new research has been focused on a small-spot SLA. Representative of such efforts is the RMPD, a new manufacturing technology in micro-engineering developed by microTEC. ${ }^{20}$ Similar to SLA, a controlled laser beam moves 


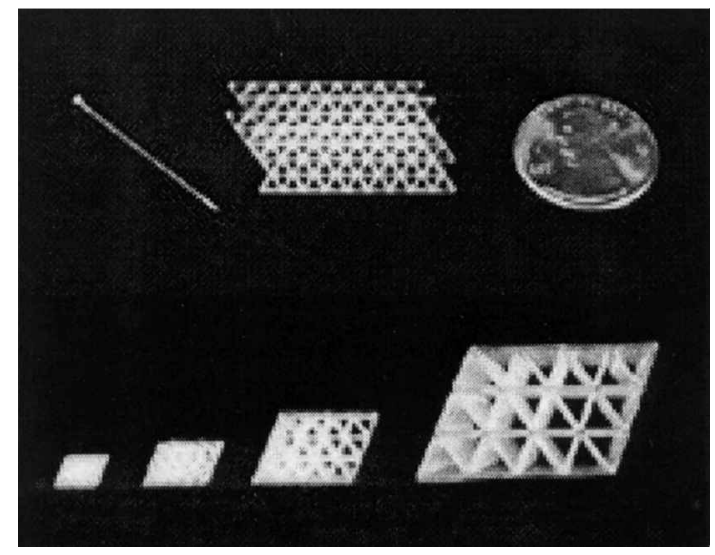

FIG. 2. MGI is using stereolithography to build concept-verification models of its tensegrity structures. ${ }^{24}$

across a specific area structures point by point and, in this manner, hardens the liquid photopolymer by photopolymerization. Typical materials used are acrylics and epoxies, but it is expected that the range of materials will be extended to include metals, ceramics, and other suitable composites. Components are built up in steps of less than $1 \mu \mathrm{m}$ thick and a resolution finer than $10 \mu \mathrm{m}$. Current technologies limit the product size to a maximum size of $35 \mathrm{~mm}^{3}$.

\section{Droplet deposition}

In droplet deposition, ${ }^{25-27}$ molten droplets deposited on the working area will soften the material of the previous layer and then solidify, joining the droplets to the previous layer. Unfilled areas may be filled with a soluble molten wax to the same thickness to act as the support material for next layer. When all layers have been deposited, the object is removed from the platform and support materials are removed by dissolving them using appropriate techniques. Examples of machines using such method include Fraunhofer's multi-phase jet solidification (MJS), Stratasys's fused deposition modeling (FDM), 3 D System's multi-jet modeling (MJM), and Sanders's model maker. ${ }^{7}$

3D honeycomb porous alumina ceramic structures for bone implants have been fabricated using indirect route where a polymeric mold is first created using FDM. ${ }^{27}$ The mold was then infiltrated with ceramic slurry, dried, and subjected to a binder burn-out and sintering cycle. 3D honeycomb structures with $33 \%$

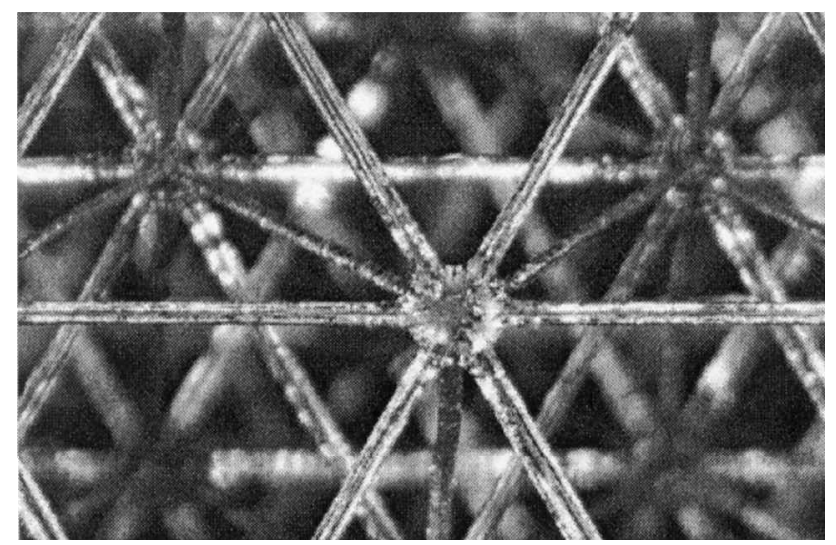

FIG. 3. The struts of the MGI biomimetic scaffold, which was built using a small-spot laser stereolithography system, measure less than $70 \mu \mathrm{m}$ in diameter. ${ }^{24}$ 


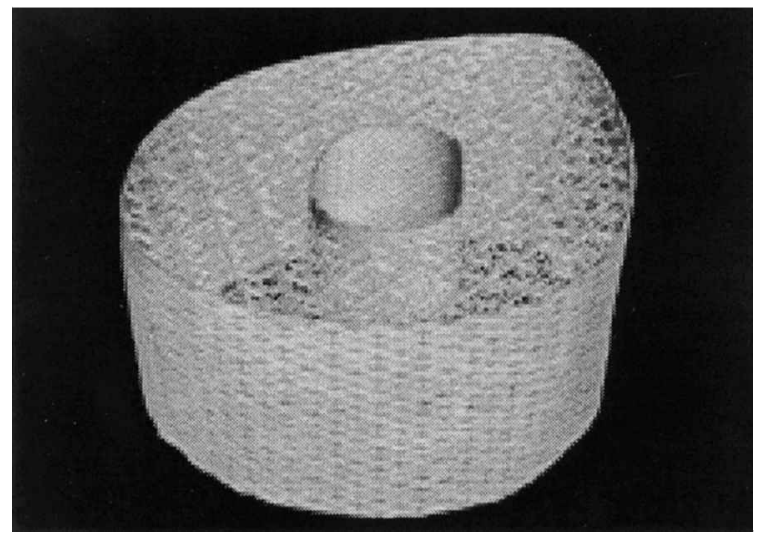

FIG. 4. A bone graft insert, made of a titanium tensegrity mesh, would be strong enough to support a person's weight and, at the same time, porous enough to encourage bone growth into the structure. ${ }^{24}$

and $50 \%$ total pore volumes were fabricated with pore sizes varying from 300 to $750 \mu \mathrm{m}$ where the pores are interconnected in all directions.

Researchers $^{28}$ at the National University of Singapore have fabricated 3D scaffold using PCL and PCL-HA composite filaments with a FDM 3D Modeler from Stratasy Inc. The filaments were extruded through a 0.41$\mathrm{mm}(0.016$ inches $)$ nozzle and a $0.25-\mathrm{mm}(0.010$ inches $)$ nozzle to construct thin square wafers $(25.4 \times 25.4 \times$ $3 \mathrm{~mm})$, cubes $(5 \times 5 \times 5 \mathrm{~mm})$, and knee meniscus. ${ }^{29}$ Scaffolds of porosity $50-80 \%$ were produced by configuring various build parameters, including the road width of rasters, fill gap between rasters, slice thickness, and raster angle (Fig. 5). The effects of scaffold morphology and culture conditions on cell behavior of an in vitro osteoblast-scaffold culture system before osteoblast transplantation were studied. It has been observed that the osteoblasts attached to the scaffold surfaces continuously proliferated over a period of 3 weeks.

Sander Prototype $\mathrm{e}^{30,31}$ has announced a program in conjunction with the University of Michigan to develop a RP machine based on its ModelMaker technology that will be capable of making patterns for use in repairing bone defects resulting from trauma, oncologic surgery, and congenital disorders. The goal of the project is to develop methods for making 3D scaffolds for skeletal reconstruction out of either bioceramics or biopolymers ${ }^{30,32-34}$ (Figs. 6 and 7).

\section{DISCUSSIONS}

\section{Materials}

Each RP technique is a process based on the unique property of the raw materials applied and the particular interaction between the materials and the energy. For instance, SLA is based on the photopolymer-

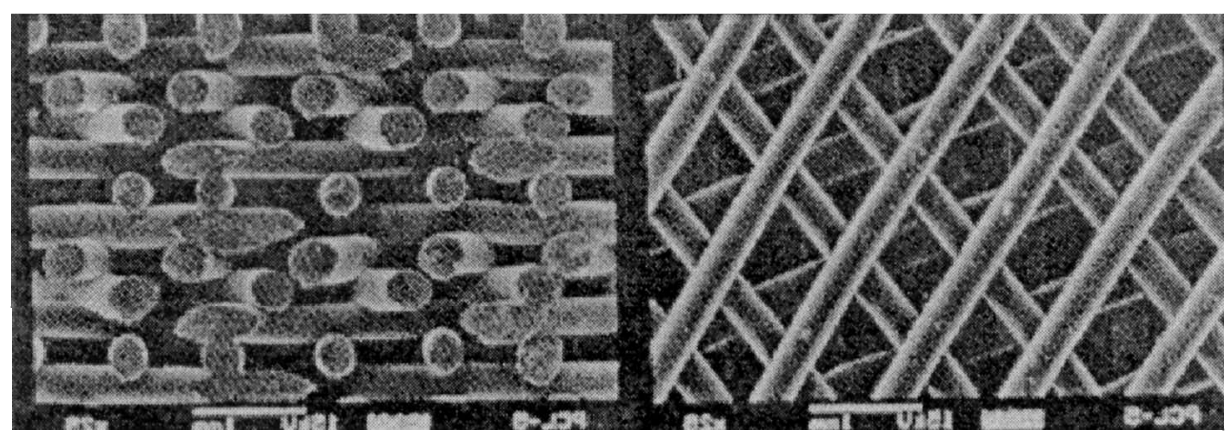

FIG. 5. Top view and cross-section of a PLC scaffold by FDM with a 0/72/144/36/108 lay-down pattern. 


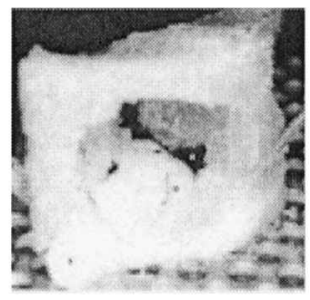

(a)

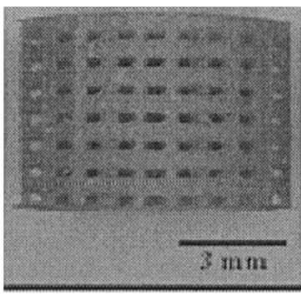

(b)

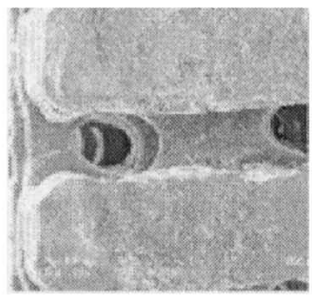

(c)

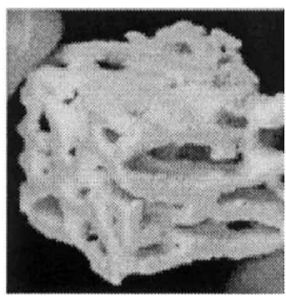

(d)

FIG. 6. Examples of scaffold fabrication from bioceramics. (a) Orbital floor scaffold made directly from patient CT data. (b) HA scaffold with regular designed pore structure. (c) SEM showing channels from scaffold in b. (d) Ceramic biomimetic scaffold made directly from micro-CT image of human trabecular bone microstructure..$^{30,33}$

ization of liquid monomer with a UV laser beam (with a low power density), while SLS is based on the melting-binding of powder with a $\mathrm{CO}_{2}$ laser (with a high power density). However, most materials used in the commercialized RP systems are so far neither biocompatible nor biodegradable. In order to create TE scaffolds by RP techniques, it is desirable that the current materials be replaced by biomaterials that can satisfy the biocompatibility and biodegradability requirements as mentioned in Part I of this article. Here, the possible issues and potential resolution methods of biomaterials in RP process are discussed.

The thermal degradation of materials in the RP processing is a major problem that should be addressed. Masanori Kikuchi et al. ${ }^{35,36}$ reported that the molecular weight of copolymer poly-L-lactide (CPLA) decreased from 100,000 to 10,000 or less due to high-temperature hydrolysis reaction when it was heated at $180^{\circ} \mathrm{C}\left(453^{\circ} \mathrm{K}\right)$ for $30 \mathrm{~min}$ in a drying oven and subsequently mixed with calcium phosphate for $15 \mathrm{~min}$. However, it was also found that thermal degradation was not obvious when the composites are prepared with minutes of thermal kneading. Based on this finding, the authors are of the opinion that CPLA will probably degrade in the chamber of SLS, while it may not do so in an extruder of the FDM.

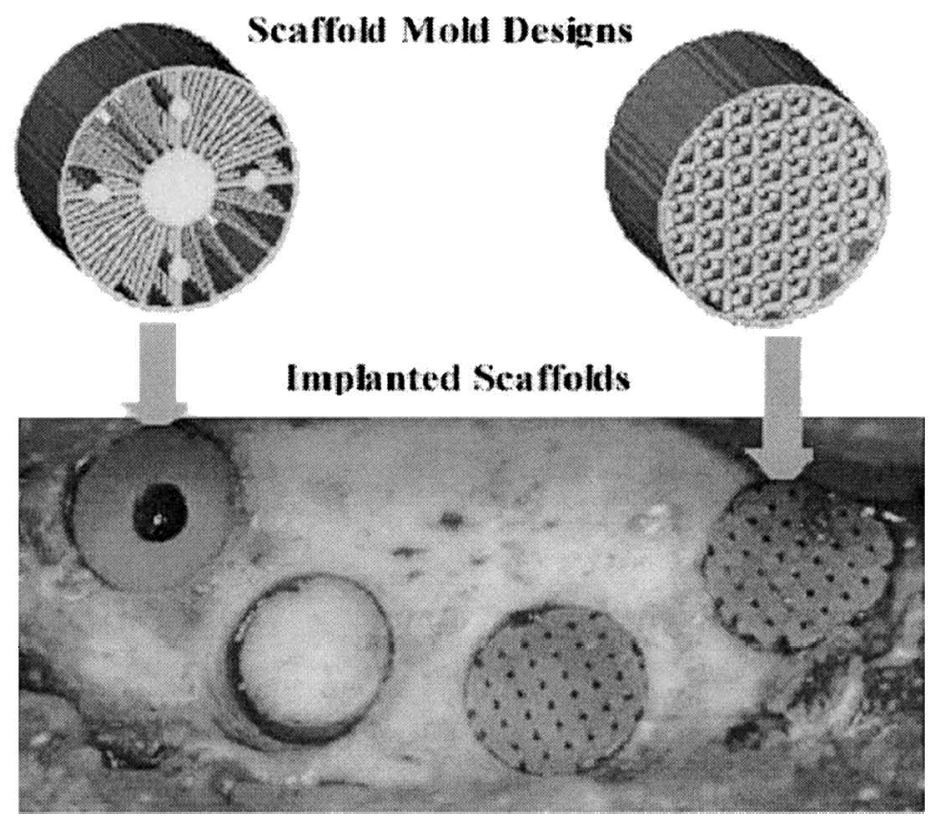

FIG. 7. Example of minipig craniofacial model used to test scaffold/biologic factor. This example shows the initial image-based scaffold design translated into fabricated scaffolds and implanted into a minipig mandible defect model. ${ }^{30,34}$ (C) 2000 Munksgaard International Publishers Ltd., Copenhagen, Denmark. 
The potential of the photopolymer-based RP approaches depends on the development of photopolymerizable, biocompatible, and biodegradable liquid polymer, which can fulfill the requirements of TE. John and Morita ${ }^{37}$ have prepared lactide-based polydepsipeptide polymer networks and cross-linked beads by UV photopolymerization of acrylated poly (L-lactic acid-co-glycolic acid-co-L-serine). These materials have been developed for use as polymer scaffolds in tissue engineering, cell encapsulation, and injectable drug delivery, which have ligand-immobilizable and biodegradable characteristics. The lactic acid/serine polymer networks were produced by irradiation by a $100-\mathrm{W}$ medium-pressure mercury ultraviolet source at an intensity of $10 \mathrm{~mW} / \mathrm{cm}^{2}$. Elisseeff et al. ${ }^{38}$ used an 8 -W LWUV lamp for $5 \mathrm{~min}$ to polymerize poly (L-lacitc acid-co-L-aspartic acid). The introduction of lysine in PLA also introduced a functional group for attachment of bioactive peptides ${ }^{37}$ Photopolymerizable, biocompatible, and water-soluble polymers such as polyethylene glycol tetraacrylate (MW 18,500) can be photopolymerized by an argon laser under biologically compatible conditions using an initiator such as triethanolamine, $N$-vinylpyrollidone, and eosin Y. ${ }^{15}$ Similar photopolymerizable macromers having a poly(ethylene glycol) central block, extended with hydrolyzable oligomers such as oligo(D,L-lactic acid) or oligo(glycolic acid) and terminated with acrylate groups, may be used. ${ }^{15}$

Preprocessing is also an issue to be considered. Certain shapes for these biodegradable materials, such as filament for FDM and micropowder for 3DP and SLS, are not available currently commercially available. The spinning of filament and grinding of powder become critical to the TE scaffold building, as the chemical and mechanical properties of materials can change during preprocessing. Giordano ${ }^{39}$ re- $^{-}$ ported that the yield of milling for PLA is low. At first they used a Waring blender to mill the liquid nitrogen-chilled PLA granules, which was supplied as granules of approximately $3 \mathrm{~mm}$ in diameter, to $75-150 \mu \mathrm{m}$, but the yield using this device reached only about 5\% of the starting mass. An Ultra Centrifugal Mill was employed to improve the yield to approximately $30 \%$ of the starting mass. Powders are stored at all times before and after milling in a vacuum at $-30 \mathrm{in} \mathrm{Hg}$ to prevent hydrolytic degradation from atmospheric humidity.

\section{The smallest feature}

The feasibility of TE scaffold fabrication also depends on the smallest feature that RP machines can build, because the scaffold is a matrix with numerous interconnected channels of less than $500 \mu \mathrm{m}$ in width.

The smallest feature depends on uncontrolled machine-specific parameters, the working principle of RP machines, as well as controlled parameters, such as geometry-specific parameters and material properties for creation of fine features. The laser spot size is the critical machine-specific parameters of SLA and SLS, whereas the nozzle diameter is the counterpart of FDM, Model Maker, and 3DP. Also, powder size is an important material parameter that affects the small features produced by powder-based RP methods (SLS and 3DP). For SLS and SLA, the Gaussian energy distribution of the laser beam makes it difficult to sinter a clear or sharp boundary.

Chua et al. ${ }^{9}$ and Johnson ${ }^{40}$ used the ultimate resolution to describe the resolution limits for RP technology (Table 1). The photopolymerization has the highest resolution, or $1.33 \times 10^{7}$ elements per $\mathrm{mm}^{3}$, which is believed to have the best potential for improving resolution. ${ }^{9}$

Table 1. Rapid Prototyping Process Performance

\begin{tabular}{lrrc}
\hline & \multicolumn{2}{c}{ Resolution (elements $\left.\mathrm{mm}^{-3}\right)$} & \\
\cline { 2 - 3 } RP processes & Present & Ultimate & $\begin{array}{c}\text { Surface roughness } \\
(\mu \mathrm{m} \text { r.m.s })\end{array}$ \\
\hline Laser curing & 3,152 & $13,300,000$ & 0.10 \\
Masked lamp & 69 & 97,500 & 4.22 \\
Sheet lamination & 1,907 & 200,000 & - \\
Laser sintering & 211 & 244,000 & $1.3-3.0$ \\
Droplet depositing & 15,200 & 157,000 & $1.6-2$ \\
Adhesive bonding & 1,900 & 244,000 & $6.6-15$ \\
\hline
\end{tabular}


Table 2. The Sequence of the Difficulty of

Trapped Materials Removal

\begin{tabular}{lll}
\hline & \multicolumn{1}{c}{ Trapped materials } & \multicolumn{1}{c}{ RP process } \\
\hline 1 & No support & FDM \\
2 & Liquid & SLA, MicroTec \\
3 & Water-soluble solid & SGC, Model Maker \\
4 & Powder & SLS, 3DP \\
5 & Water-insoluble solid & LOM \\
\hline
\end{tabular}

The major advantage of RP on TE scaffold is its ability to overcome the problem of uncontrollable microstructure and feasibility issues of complex 3D structure of traditional methods. Another important feature of RP in TE is its freeform fabrication capability of achieving prototypes or products rather than RP.

\section{Processability and trapped materials}

The geometry of TE scaffolds should be a matrix with numerous internal interconnected channels or pores of less than $500 \mu \mathrm{m}$ that is shaped by support materials or trapped materials which have to be removed after fabrication. Trapped materials can be classified into four classes according to their physical state and different RP methods: (1) liquid (SLA, MicroTec), (2) water-soluble solid (SGC, Model Maker), (3) water-insoluble solid (LOM), and (4) powder (SLS, 3DP). It is proven that the support can be eliminated in the FDM process because the continuity of the filament holds the material from falling into the void before solidification, provided that the size of the void is sufficiently small. For instance, the scaffold structure can be built without any support in the voids sized 100-500 $\mu \mathrm{m}$. In creating special scaffolds, the liquid trapped in SLA or loose powder in SLS or 3DP is not support per se (i.e., generated intentionally) but happened as part of the process. The surface roughness and the aggregation or viscosity of support materials affect the efficiency of removal of such trapped materials. The laser curing method has the smallest surface roughness, at $\mathrm{Ra} 0.10$, whereas the adhesive bonding has the biggest, at Ra6.6-15. ${ }^{9}$ Experiment results show that, the smoother the surface generated, the lower the viscosity of materials, the easier the removal of trapped materials. A list of the methods with trapped materials is given in Table 2 in order of difficulty of removal.

Table 3. The Advantages and Limitations of Rapid Prototyping in the Tissue-Engineering Scaffold

Advantage Limitation

Sheet lamination (LOM)

Adhesion bonding (3DP)

Laser sintering (SLS)

Photopolymerization

(SLA, SGC, MicroTec)

Droplet deposition (FDM)

Model maker
More materials choice; Low heat effect on raw powder

Relative higher part strength More materials choice.

Relative easy to remove support materials; relative easy to achieve small feature.

No materials trapped in the scaffold; easy to achieve $\sim 100 \mu \mathrm{m}$ scaffold feature

Easy to achieve $\sim 100 \mu \mathrm{m}$ or smaller scaffold feature
Materials trapped in small inner holes is impossible to be removed

Materials trapped in small inner holes is difficult to be removed

Materials trapped in small inner holes is difficult to be removed; biodegradable materials maybe will degrade in the chamber

Limited by the development of photopolymerizable and biocompatible, biodegradable liquid polymer material

Relative regular structure; anisotropy between XY and $\mathrm{Z}$ direction; High heat effect on raw material

High heat effect on raw material; difficult to change materials without manufacturer's cooperation 


\section{CONCLUSION}

Each RP process has its own advantages and disadvantages in building TE scaffolds, as summarized in Table 3. For example, in terms of microstructure manufacturability, MicroTec, at a very fine resolution, which is unique in construction and connection technology, shows the best potential. In terms of trapped material removal, FDM has a major advantage as no support material is needed during the fabrication of scaffolds. Conversely, LOM has a major problem in producing microchannels since sheet material is preprocessed before lamination. In terms of suitability of material, SLS and 3DP processes have more diversity, as both polymer and ceramic powders are formable on current systems. In addition, the property of materials such as molecular weight have to be kept almost constant even after processed by RP. Processes without heating or with low temperature heating, such as 3DP, stand a better chance by minimizing the heating effect on materials. SLS has a major advantage that no binder is needed in the process, compared with 3DP.

The most likely direction of development in using RP on TE scaffold will lead to the development of a specialized machine for TE manufacture. Once the application is narrowed to scaffold fabrication, the material and microstructure capabilities become much more important than the part size capacity and building speed, which, however, are major concerns for industry-oriented systems.

\section{REFERENCES}

1. U.S. Scientific Registry for Organ Transplantation and the Organ Procurement and Transplant Network. Annual Report. Richmond VA: UNOS, 1990.

2. Vacanti, J., and Vacanti, C. The challenge of tissue engineering. In: Lanza R.P., Langer, R., Chick, W.L., eds. Principles of Tissue Engineering. Austin, TX: Academic Press, 1997, pp. 1-6.

3. Cohen, S., Bano, M.C., Cima L.G., et al. Design of synthetic polymeric structures for cell transplantation and tissue engineering. Clin. Mater. 13, 3, 1993.

4. Langer, R. and Vacanti, J.P. Tissue Engineering. Science 260, 920, 1993.

5. Cima, L.G., Vacanti, J.P., Vacanti, C., et al. Tissue engineering by cell transplantation using degradable polymer substrates. J. Biomech. Eng. 113, 143, 1991.

6. Cima, L.B., Ingber, C., Vacanti, J.P., et al. Hepatocyte culture on biodegradable polymeric substrates. Biotechnol. Bioeng. 38, 145, 1991.

7. Chua, C.K., and Leong, K.F. Rapid Prototyping: Principles and Applications in Manufacturing. New York: Wiley, 1997.

8. Yang, S.F., Du, Z.H., Leong K.F., et al. The design of scaffolds for use in tissue engineering. Part I. Traditional factors. Tissue Eng. 7, 679, 2001.

9. Chua, C.K., Chou, S.M., and Wong, T.S. A study of the state-of-the-art rapid prototyping technologies. Int. J. Adv. Manufact. Technol. 14, 146, 1998.

10. Steidle, C., Klosterman, D., Osborne, N., et al. Automated fabrication of nonresorbable bone implants using laminated object manufacturing (LOM). Presented at the ' 98 SFF, 1998.

11. Steidle, C., Klosterman, D., Chartoff R., et al. Automated fabrication of custom bone implants using rapid prototyping. Presented at the 44th International SAMPE Symposium and Exhibition, Long Beach, California, May 1999.

12. Cima, M.J., Sachs, E., Cima, L.G., et al. Computer-driven microstructures by 3D printing: bio- and structural materials. Presented at the '94 SFF, Austin, TX, August 8-10, 1994.

13. Wu, B.M., Borland, S.W., Giordano, R.A., et al. Solid free form fabrication of drug delivery devices. J. Controlled Release 40, 77, 1996.

14. TheriForm ${ }^{\mathrm{TM}}$ technology [On-line]. Available: www.therics.com/theriform.html.

15. Cima, L.G., and Cima, M.J. Preparation of medical devices by solid free-form fabrication methods. U.S. patent $5,490,962$.

16. Giordano, R.A., Wu, B.M., Borland, S.W., et al. Mechanical properties of dense polylactic acid structures fabricated by three-dimensional printing. J. Biomater. Sci. Polym. Ed. 8, 63, 1996.

17. Lee, G., and Barlow, J.W. Selective laser sintering of bioceramic materials for implants. Presented at the ' 93 SFF, Austin, TX, August 9-11, 1993.

18. Lee, G., Barlow, J.W., Fox, W.C., et al. Biocompatibility of SLS-formed calcium phosphate implants. Presented at the '96 SFF, Austin, TX, August 12-14, 1996. 
19. Vail, N.K., Swain, L.D., Fox, W.C., et al. Materials for biomedical applications. Presented at the '98 SFF, Austin, TX, August 10-12, 1998.

20. Gesellschaft für MikrotechnologiembH. Microsystems production with rapid micro product development (RMPD) [On-line]. Available: www. microtec-d.com/engl.htm.

21. Langton, C.M., Whitehead, M.A., Langton, D.K., et al. Development of a cancellous bone structural model by stereolithography for ultrasound characterisation of the calcaneus. Med. Eng. Phys. 19, 599, 1997.

22. Chu, G.T.-M., Brady, G.A., Miao, W., et al. Ceramic SFF by direct and indirect stereolithography. Presented at Materials Research Society, Fall Meeting, November 30-December 3, 1999, Boston, MA, pp. 119-123.

23. Rapid prototyping helps duplicate the structure of life. Rapid Prototyping Rep. 9, 1, 1999.

24. Molecular Geodesics, Inc. Innovation through biological mimicry [On-line]. Available: www.molecgeodesics. com/contactUs.html.

25. Pekin, S., Koehl, D., and Zangvil, A. A new HAPEX-like biocomposite that can be shaped by fused deposition of materials (FDM). Presented at the '99 SFF, Austin, TX, August 9-11, 1999.

26. Pekin, S., and Zangvil, A. Effect of powder characteristics on the sintering of hydroxyapatite feedstocks that can be shaped by FDM. Presented at the '99 SFF, Austin, TX, August 9-11, 1999.

27. Bose, S., Avila, M., and Bandyopadhyay, A. Processing of bioceramic implants via fused deposition process. Presented at the '98 SFF, Austin, TX, August 10-12, 1998.

28. Zein, I., Hutmacher, D.W., Schantz, J.T., et al. Processing of 3D scaffolds by fused deposition modeling. Presented at the International Workshop on Advances in Materials Science and Technology, Singapore, April 2000.

29. Schantz, J.T., Hutmacher, D.W., Zein, I., et al. A study of human osteoblasts on poly(caprolactone)-scaffolds. Presented at the International Workshop on Advances in Materials Science and Technology, Singapore, April 2000.

30. Hollister, S.J. University of Michigan White Paper BRP proposal: systems integration for skeletal engineering. [Online]. Available. www.bme.umich.edu/skelegen/.

31. Sanders Prototype. Development opportunities: Sanders seeking partners for medical ModelMaker. Rapid Prototyping Rep. 9, 7, 1999.

32. Hollister, S.J., Levy, R.A., Chu, T.-M.G., et al. Design and manufacture of an orbital floor scaffold using image processing and rapid prototyping. Presented at the American Society of Mechanical Engineers (ASME) Bioengineering Conference, New York, 1997.

33. Levy, R.A., Chu, T.M.G., Halloran, J.W., et al. CT-generated porous Hydroxyapatite orbital floor prothesis as a prototype bioimplant. Am. J. Neuroradiol. 18, 1522, 1997.

34. Hollister, S.J., Chu, T.M., Levy, R.A., et al. An image-based approach to designing and manufacturing craniofacial scaffolds. Int. J. Oral Maxillofac. Surg. 29, 67, 2000.

35. Kikuchi, M., Cho, S., Tanaka, J., et al. Preparation and biocompatibility of $\alpha \beta$-tricalcium-phosphate/copolymerized-poly-L-lactide composite. J Jpn Soc Powder Metallurgy 45, 36, 1999.

36. Kikuchi, M., Suecsugu, Y., Tanaka, J., et al. Preparation and mechanical properties of calcium phosphate/copolyL-lactide composite. J. Mater. Sci. 8, 361, 1997.

37. John, G., and Morita, M. Synthesis and characterization of photo-cross-linked networks based on L-lactide/serine copolymers. Macromolecules 32, 1853, 1999.

38. Elisseeff, J., Anseth, K., Langer, R., et al. Synthesis and characterization of photo-cross-linked polymers based on poly(L-lacitc acid-co-L-aspartic acid). Macromolecules 30, 2182, 1997.

39. Giordano, R.A., Wu, B.M., Borland, S.W., et al. Mechanical properties of dense polylactic acid structures fabricated by three dimensional printing. J. Biomater. Sci. Polym. Ed. 8, 63, 1996.

40. Johnson, J.L. Principles of complete automated fabrication. Paletino, 1994.

Address reprint requests to: Kah-Fai Leong, M.S.E., M.S.M.E.

Design Research Center School of Mechanical and Production Engineering Nanyang Technological University

Nanyang Avenue Singapore, 639798

E-mail: mkfleong@ntu.edu.sq 